\title{
Symmetries and invariants of twisted quantum algebras and associated Poisson algebras
}

\author{
A. I. Molev and E. Ragoucy
}

\begin{abstract}
We construct an action of the braid group $B_{N}$ on the twisted quantized enveloping algebra $\mathrm{U}_{q}^{\prime}\left(\mathfrak{o}_{N}\right)$ where the elements of $B_{N}$ act as automorphisms. In the classical limit $q \rightarrow 1$ we recover the action of $B_{N}$ on the polynomial functions on the space of upper triangular matrices with ones on the diagonal. The action preserves the Poisson bracket on the space of polynomials which was introduced by Nelson and Regge in their study of quantum gravity and rediscovered in the mathematical literature. Furthermore, we construct a Poisson bracket on the space of polynomials associated with another twisted quantized enveloping algebra $\mathrm{U}_{q}^{\prime}\left(\mathfrak{s p}_{2 n}\right)$. We use the Casimir elements of both twisted quantized enveloping algebras to re-produce some well-known and construct some new polynomial invariants of the corresponding Poisson algebras.
\end{abstract}

Preprint LAPTH-1174/07

School of Mathematics and Statistics

University of Sydney, NSW 2006, Australia

alexm@maths.usyd.edu.au

LAPTH, Chemin de Bellevue, BP 110

F-74941 Annecy-le-Vieux cedex, France

ragoucy@lapp.in2p3.fr 


\section{Introduction}

Deformations of the commutation relations of the orthogonal Lie algebra $\mathfrak{o}_{3}$ were considered by many authors. The earliest reference we are aware of is Santilli [28]. Such deformed relations can be written as

$$
q X Y-Y X=Z, \quad q Y Z-Z Y=X, \quad q Z X-X Z=Y .
$$

More precisely, regarding $q$ as a formal variable, we consider the associative algebra $\mathrm{U}_{q}^{\prime}\left(\mathfrak{o}_{3}\right)$ over the field of rational functions $\mathbb{C}(q)$ in $q$ with the generators $X, Y, Z$ and defining relations (1.1). From an alternative viewpoint, relations (1.1) define a family of algebras depending on the complex parameter $q$. The same algebras were also defined by Odesskii [26], Fairlie [9] and Nelson, Regge and Zertuche [23]. Putting $q=1$ in (1.1) we get the defining relations of the universal enveloping algebra $\mathrm{U}\left(\mathfrak{o}_{3}\right)$. The algebra $\mathrm{U}_{q}^{\prime}\left(\mathfrak{o}_{3}\right)$ should be distinguished from the quantized enveloping algebra $\mathrm{U}_{q}\left(\mathfrak{o}_{3}\right) \cong \mathrm{U}_{q}\left(\mathfrak{s l}_{2}\right)$. The latter is a deformation of $\mathrm{U}\left(\mathfrak{o}_{3}\right)$ in the class of Hopf algebras; see e.g. Chari and Pressley [4, Section 6].

Introducing the generators

$$
x=\left(q-q^{-1}\right) X, \quad y=\left(q-q^{-1}\right) Y, \quad z=\left(q-q^{-1}\right) Z,
$$

we can write the defining relations of $\mathrm{U}_{q}^{\prime}\left(\mathfrak{o}_{3}\right)$ in the equivalent form

$$
\begin{aligned}
& q x y-y x=\left(q-q^{-1}\right) z, \\
& q y z-z y=\left(q-q^{-1}\right) x, \\
& q z x-x z=\left(q-q^{-1}\right) y .
\end{aligned}
$$

Note that the element $x^{2}+q^{-2} y^{2}+z^{2}-x y z$ belongs to the center of $\mathrm{U}_{q}^{\prime}\left(\mathfrak{o}_{3}\right)$. This time, putting $q=1$ into the defining relations we get the algebra of polynomials $\mathbb{C}[x, y, z]$. Moreover, this algebra can be equipped with a Poisson bracket in a usual way

$$
\{f, g\}=\left.\frac{f g-g f}{1-q}\right|_{q=1} .
$$

Thus, $\mathbb{C}[x, y, z]$ becomes a Poisson algebra with the bracket given by

$$
\{x, y\}=x y-2 z, \quad\{y, z\}=y z-2 x, \quad\{z, x\}=z x-2 y .
$$

These formulas are contained in the paper by Nelson, Regge and Zertuche [23]. In the classical limit $q \rightarrow 1$ the central element $x^{2}+q^{-2} y^{2}+z^{2}-x y z$ becomes the Markov polynomial $x^{2}+y^{2}+z^{2}-x y z$ which is an invariant of the bracket. The Poisson bracket 
(1.2) was re-discovered by Dubrovin [8, where $x, y, z$ are interpreted as the entries of $3 \times 3$ upper triangular matrices with ones on the diagonal (the Stokes matrices)

$$
\left(\begin{array}{lll}
1 & x & y \\
0 & 1 & z \\
0 & 0 & 1
\end{array}\right)
$$

For an arbitrary $N$ the twisted quantized enveloping algebra $\mathrm{U}_{q}^{\prime}\left(\mathfrak{o}_{N}\right)$ was introduced by Gavrilik and Klimyk [11] which essentially coincides with the algebra of Nelson and Regge [20]. Both in the orthogonal and symplectic case the twisted analogues of the quantized enveloping algebras were introduced by Noumi [24] using an $R$ matrix approach. In the orthogonal case this provides an alternative presentation of $\mathrm{U}_{q}^{\prime}\left(\mathfrak{o}_{N}\right)$. The finite-dimensional irreducible representations of the algebra $\mathrm{U}_{q}^{\prime}\left(\mathfrak{o}_{N}\right)$ were classified by Iorgov and Klimyk [14].

In the limit $q \rightarrow 1$ the twisted quantized enveloping algebra $\mathrm{U}_{q}^{\prime}\left(\mathfrak{o}_{N}\right)$ gives rise to a Poisson algebra of polynomial functions $\mathcal{P}_{N}$ on the space of Stokes matrices. The corresponding Poisson bracket was given in [20]. The same bracket was also found by Ugaglia [29], Boalch [1] and Bondal [2, 3]. This Poisson structure was studied by Ping Xu [30] in the context of Dirac submanifolds, while Chekhov and Fock [6] considered it in relation with the Teichmüller spaces. A quantization of the Poisson algebra of Stokes matrices leading to the algebra $\mathrm{U}_{q}^{\prime}\left(\mathfrak{o}_{N}\right)$ was constructed by Ciccoli and Gavarini [7] in the context of the general "quantum quality principle"; see also Gavarini [10]. It was shown by Odesskii and Rubtsov [27] that the Poisson bracket on the space of Stokes matrices is essentially determined by its Casimir elements.

Automorphisms of both the algebra $\mathrm{U}_{q}^{\prime}\left(\mathfrak{o}_{N}\right)$ and the Poisson bracket on $\mathcal{P}_{N}$ were given in [21, 22], although the explicit group relations between them were only discussed in the classical limit for $N=6$. An action of the braid group $B_{N}$ on the Poisson algebra $\mathcal{P}_{N}$ was given by Dubrovin [8] and Bondal [2].

In this paper we produce a "quantized" action of $B_{N}$ on the twisted quantized enveloping algebra $\mathrm{U}_{q}^{\prime}\left(\mathfrak{o}_{N}\right)$, where the elements of $B_{N}$ act as automorphisms. Since $\mathrm{U}_{q}^{\prime}\left(\mathfrak{o}_{N}\right)$ is a subalgebra of the quantized enveloping algebra $\mathrm{U}_{q}\left(\mathfrak{g l}_{N}\right)$, one could expect that Lusztig's action of $B_{N}$ on $\mathrm{U}_{q}\left(\mathfrak{g l}_{N}\right)$ (see [16]) leaves the subalgebra $\mathrm{U}_{q}^{\prime}\left(\mathfrak{o}_{N}\right)$ invariant. However, this turns out not to be true, and the action of $B_{N}$ on $\mathrm{U}_{q}^{\prime}\left(\mathfrak{o}_{N}\right)$ can rather be regarded as a $q$-version of the natural action of the symmetric group $\mathfrak{S}_{N}$ on the universal enveloping algebra $\mathrm{U}\left(\mathfrak{o}_{N}\right)$.

The relationship between $\mathrm{U}_{q}^{\prime}\left(\mathfrak{o}_{N}\right)$ and the Poisson algebra $\mathcal{P}_{N}$ can also be exploited in a different way. Some families of Casimir elements of $\mathrm{U}_{q}^{\prime}\left(\mathfrak{o}_{N}\right)$ were produced by Noumi, Umeda and Wakayama [25], Gavrilik and Iorgov [12] and Molev, Ragoucy and Sorba [19]. This gives the respective families of Casimir elements of the Poisson 
algebra. We show that the Casimir elements of [19] specialize precisely to the coefficients of the characteristic polynomial of Nelson and Regge [22]. This polynomial was re-discovered by Bondal [2] who also produced an algebraically independent set of generators of the subalgebra of invariants of the Poisson algebra $\mathcal{P}_{N}$. Furthermore, using [12] and [25] we obtain new Pfaffian type invariants and analogues of the Gelfand invariants.

In a similar manner, we use the twisted quantized enveloping algebra $\mathrm{U}_{q}^{\prime}\left(\mathfrak{s p}_{2 n}\right)$ associated with the symplectic Lie algebra $\mathfrak{s p}_{2 n}$ to produce a symplectic version of the above results. First, we construct a Poisson algebra associated with $\mathrm{U}_{q}^{\prime}\left(\mathfrak{s p}_{2 n}\right)$ by taking the limit $q \rightarrow 1$ and thus produce explicit formulas for the Poisson bracket on the corresponding space of matrices. Then using the Casimir elements of $\mathrm{U}_{q}^{\prime}\left(\mathfrak{s p}_{2 n}\right)$ constructed in [19], we produce a family of invariants of the Poisson algebra analogous to [2] and [22]. We also show that some elements of the braid group $B_{2 n}$ preserve the subalgebra $\mathrm{U}_{q}^{\prime}\left(\mathfrak{s p}_{2 n}\right)$ of $\mathrm{U}_{q}\left(\mathfrak{g l}_{2 n}\right)$. We conjecture that there exists an action of the semi-direct product $B_{n} \ltimes \mathbb{Z}^{n}$ on $\mathrm{U}_{q}^{\prime}\left(\mathfrak{s p}_{2 n}\right)$ analogous to the $B_{N^{-}}$action on $\mathrm{U}_{q}^{\prime}\left(\mathfrak{o}_{N}\right)$. We show that the conjecture is true for $n=2$.

This work was inspired by Alexei Bondal's talk at the Prague's conference ISQS 2006. We would like to thank Alexei for many stimulating discussions. The financial support of the Australian Research Council is acknowledged. The second author is grateful to the University of Sydney for the warm hospitality during his visit.

After we prepared the first version of our paper we learned of a recent preprint by L. Chekhov [5] where he produces (without detailed proofs) an action of the braid group $B_{N}$ on $\mathrm{U}_{q}^{\prime}\left(\mathfrak{o}_{N}\right)$ equivalent to ours.

\section{Braid group action}

We start with some definitions and recall some well-known results. Let $q$ be a formal variable. The quantized enveloping algebra $\mathrm{U}_{q}\left(\mathfrak{g l}_{N}\right)$ is an algebra over $\mathbb{C}(q)$ generated by elements $t_{i j}$ and $\bar{t}_{i j}$ with $1 \leqslant i, j \leqslant N$ subject to the relations

$$
\begin{aligned}
t_{i j} & =\bar{t}_{j i}=0, \quad 1 \leqslant i<j \leqslant N, \\
t_{i i} \bar{t}_{i i} & =\bar{t}_{i i} t_{i i}=1, \quad 1 \leqslant i \leqslant N, \\
R T_{1} T_{2} & =T_{2} T_{1} R, \quad R \bar{T}_{1} \bar{T}_{2}=\bar{T}_{2} \bar{T}_{1} R, \quad R \bar{T}_{1} T_{2}=T_{2} \bar{T}_{1} R .
\end{aligned}
$$

Here $T$ and $\bar{T}$ are the matrices

$$
T=\sum_{i, j} t_{i j} \otimes E_{i j}, \quad \bar{T}=\sum_{i, j} \bar{t}_{i j} \otimes E_{i j},
$$


which are regarded as elements of the algebra $\mathrm{U}_{q}\left(\mathfrak{g l}_{N}\right) \otimes$ End $\mathbb{C}^{N}$, the $E_{i j}$ denote the standard matrix units and the indices run over the set $\{1, \ldots, N\}$. Both sides of each of the $R$-matrix relations in (2.1) are elements of $\mathrm{U}_{q}\left(\mathfrak{g l}_{N}\right) \otimes$ End $\mathbb{C}^{N} \otimes$ End $\mathbb{C}^{N}$ and the subscripts of $T$ and $\bar{T}$ indicate the copies of End $\mathbb{C}^{N}$, e.g.,

$$
T_{1}=\sum_{i, j} t_{i j} \otimes E_{i j} \otimes 1, \quad T_{2}=\sum_{i, j} t_{i j} \otimes 1 \otimes E_{i j}
$$

while $R$ is the $R$-matrix

$$
R=q \sum_{i} E_{i i} \otimes E_{i i}+\sum_{i \neq j} E_{i i} \otimes E_{j j}+\left(q-q^{-1}\right) \sum_{i<j} E_{i j} \otimes E_{j i} .
$$

In terms of the generators the defining relations between the $t_{i j}$ can be written as

$$
q^{\delta_{i j}} t_{i a} t_{j b}-q^{\delta_{a b}} t_{j b} t_{i a}=\left(q-q^{-1}\right)\left(\delta_{b<a}-\delta_{i<j}\right) t_{j a} t_{i b},
$$

where $\delta_{i<j}$ equals 1 if $i<j$, and 0 otherwise. The relations between the $\bar{t}_{i j}$ are obtained by replacing $t_{i j}$ by $\bar{t}_{i j}$ everywhere in (2.4), while the relations involving both $t_{i j}$ and $\bar{t}_{i j}$ have the form

$$
q^{\delta_{i j}} \bar{t}_{i a} t_{j b}-q^{\delta_{a b}} t_{j b} \bar{t}_{i a}=\left(q-q^{-1}\right)\left(\delta_{b<a} t_{j a} \bar{t}_{i b}-\delta_{i<j} \bar{t}_{j a} t_{i b}\right) .
$$

The braid group $B_{N}$ is generated by elements $\beta_{1}, \ldots, \beta_{N-1}$ subject to the defining relations

$$
\beta_{i} \beta_{i+1} \beta_{i}=\beta_{i+1} \beta_{i} \beta_{i+1}, \quad i=1, \ldots, N-2
$$

and

$$
\beta_{i} \beta_{j}=\beta_{j} \beta_{i}, \quad|i-j|>1 .
$$

The group $B_{N}$ acts on the algebra $\mathrm{U}_{q}\left(\mathfrak{g l}_{N}\right)$ by automorphisms; see Lusztig [16]. Explicit formulas for the images of the generators are found from [16] by re-writing the action in terms of the presentation (2.1). For any $i=1, \ldots, N-1$ we have

$$
\begin{aligned}
& \beta_{i}: t_{i i} \mapsto t_{i+1, i+1}, \quad t_{i+1, i+1} \mapsto t_{i i}, \quad t_{k k} \mapsto t_{k k} \quad \text { if } \quad k \neq i, i+1, \\
& \beta_{i}: t_{i+1, i} \mapsto q^{-1} \bar{t}_{i, i+1} t_{i i}^{2} \\
& t_{i k} \mapsto q t_{i k} t_{i+1, i} \bar{t}_{i i}-t_{i+1, k}, \quad t_{i+1, k} \mapsto q^{-1} t_{i k}, \quad \text { if } \quad k \leqslant i-1 \\
& t_{l i} \mapsto q^{-1} \bar{t}_{i, i+1} t_{l i} t_{i i}-t_{l, i+1}, \quad t_{l, i+1} \mapsto q t_{l i}, \quad \text { if } l \geqslant i+2 \\
& t_{k l} \mapsto t_{k l} \quad \text { in all remaining cases, }
\end{aligned}
$$

and

$$
\begin{aligned}
\beta_{i}: \bar{t}_{i, i+1} & \mapsto q \bar{t}_{i i}^{2} t_{i+1, i} & & \\
\bar{t}_{k i} \mapsto q{ }^{-1} t_{i i} \bar{t}_{i, i+1} \bar{t}_{k i}-\bar{t}_{k, i+1}, & & \bar{t}_{k, i+1} \mapsto q \bar{t}_{k i}, & \text { if } k \leqslant i-1 \\
\bar{t}_{i l} \mapsto q \bar{t}_{i i} \bar{t}_{i l} t_{i+1, i}-\bar{t}_{i+1, l}, & & \bar{t}_{i+1, l} \mapsto q^{-1} \bar{t}_{i l}, & \text { if } l \geqslant i+2 \\
\bar{t}_{k l} \mapsto \bar{t}_{k l} & & \text { in all remaining cases. } &
\end{aligned}
$$


Following Noumi [24] we define the twisted quantized enveloping algebra $\mathrm{U}_{q}^{\prime}\left(\mathfrak{o}_{N}\right)$ as the subalgebra of $\mathrm{U}_{q}\left(\mathfrak{g l}_{N}\right)$ generated by the matrix elements $s_{i j}$ of the matrix $S=T \bar{T}^{t}$ so that

$$
s_{i j}=\sum_{k=1}^{N} t_{i k} \bar{t}_{j k} .
$$

Equivalently, $\mathrm{U}_{q}^{\prime}\left(\mathfrak{o}_{N}\right)$ is generated by the elements $s_{i j}$ subject only to the relations

$$
\begin{gathered}
s_{i j}=0, \quad 1 \leqslant i<j \leqslant N, \\
s_{i i}=1, \quad 1 \leqslant i \leqslant N, \\
R S_{1} R^{t} S_{2}=S_{2} R^{t} S_{1} R,
\end{gathered}
$$

where $R^{t}:=R^{t_{1}}$ denotes the element obtained from $R$ by the transposition in the first tensor factor:

$$
R^{t}=q \sum_{i} E_{i i} \otimes E_{i i}+\sum_{i \neq j} E_{i i} \otimes E_{j j}+\left(q-q^{-1}\right) \sum_{i<j} E_{j i} \otimes E_{j i} .
$$

In terms of the generators, the relations (2.8) take the form

$$
\begin{aligned}
q^{\delta_{j k}+\delta_{i k}} s_{i j} s_{k l}-q^{\delta_{j l}+\delta_{i l}} s_{k l} s_{i j} & =\left(q-q^{-1}\right) q^{\delta_{j i}}\left(\delta_{l<j}-\delta_{i<k}\right) s_{k j} s_{i l} \\
& +\left(q-q^{-1}\right)\left(q^{\delta_{j l}} \delta_{l<i} s_{k i} s_{l j}-q^{\delta_{i k}} \delta_{j<k} s_{i k} s_{j l}\right) \\
& +\left(q-q^{-1}\right)^{2}\left(\delta_{l<j<i}-\delta_{j<i<k}\right) s_{k i} s_{j l},
\end{aligned}
$$

where $\delta_{i<j}$ or $\delta_{i<j<k}$ equals 1 if the subscript inequality is satisfied, and 0 otherwise. Equivalently, the set of relations can also be written as

$$
\begin{aligned}
s_{i j} s_{k l}-s_{k l} s_{i j} & =0 & & \text { if } i>j>k>l \\
s_{i j} s_{k l}-s_{k l} s_{i j} & =0 & & \text { if } i>k>l>j \\
s_{i j} s_{k l}-s_{k l} s_{i j} & =\left(q-q^{-1}\right)\left(s_{k j} s_{i l}-s_{i k} s_{j l}\right) & & \text { if } i>k>j>l \\
q s_{i j} s_{j l}-s_{j l} s_{i j} & =\left(q-q^{-1}\right) s_{i l} & & \text { if } i>j>l \\
q s_{i j} s_{i l}-s_{i l} s_{i j} & =\left(q-q^{-1}\right) s_{l j} & & \text { if } i>l>j \\
q s_{i j} s_{k j}-s_{k j} s_{i j} & =\left(q-q^{-1}\right) s_{k i} & & \text { if } \quad k>i>j .
\end{aligned}
$$

In this form the relations were given by Nelson and Regge [20]. An analogue of the Poincaré-Birkhoff-Witt theorem for the algebra $\mathrm{U}_{q}^{\prime}\left(\mathfrak{o}_{N}\right)$ was proved in [13]; see also [17, 19] for other proofs. This theorem implies that at $q=1$ the algebra $\mathrm{U}_{q}^{\prime}\left(\mathfrak{o}_{N}\right)$ specializes to the algebra of polynomials in $N(N-1) / 2$ variables. More precisely, set $\mathcal{A}=\mathbb{C}\left[q, q^{-1}\right]$ and consider the $\mathcal{A}$-subalgebra $\mathrm{U}_{\mathcal{A}}^{\prime}$ of $\mathrm{U}_{q}^{\prime}\left(\mathfrak{o}_{N}\right)$ generated by the elements $s_{i j}$. Then we have an isomorphism

$$
\mathrm{U}_{\mathcal{A}}^{\prime} \otimes_{\mathcal{A}} \mathbb{C} \cong \mathcal{P}_{N}
$$


where the action of $\mathcal{A}$ on $\mathbb{C}$ is defined via the evaluation $q=1$ and $\mathcal{P}_{N}$ denotes the algebra of polynomials in the independent variables $a_{i j}$ with $1 \leqslant j<i \leqslant N$. The elements $a_{i j}$ are respective images of the $s_{i j}$ under the isomorphism (2.12). Furthermore, the algebra $\mathcal{P}_{N}$ is equipped with the Poisson bracket $\{\cdot, \cdot\}$ defined by

$$
\{f, h\}=\left.\frac{\tilde{f} \tilde{h}-\tilde{h} \tilde{f}}{1-q}\right|_{q=1},
$$

where $f, h \in \mathcal{P}_{N}$ and $\tilde{f}$ and $\tilde{h}$ are elements of $\mathrm{U}_{\mathcal{A}}^{\prime}$ whose images in $\mathcal{P}_{N}$ under the specialization $q=1$ coincide with $f$ and $h$, respectively. Indeed, write the element $\widetilde{f} \tilde{h}-\widetilde{h} \tilde{f} \in \mathrm{U}_{\mathcal{A}}^{\prime}$ as a linear combination of the ordered monomials in the generators with coefficients in $\mathcal{A}$. Since the image of $\widetilde{f} \widetilde{h}-\widetilde{h} \widetilde{f}$ in $\mathcal{P}_{N}$ is zero, all the coefficients are divisible by $1-q$. Clearly, the element $\{f, h\} \in \mathcal{P}_{N}$ is independent of the choice of $\widetilde{f}$ and $\widetilde{h}$ and of the ordering of the generators of $\mathrm{U}_{\mathcal{A}}^{\prime}$. Obviously, (2.13) does define a Poisson bracket on $\mathcal{P}_{N}$. By definition,

$$
\left\{a_{i j}, a_{k l}\right\}=\left.\frac{s_{i j} s_{k l}-s_{k l} s_{i j}}{1-q}\right|_{q=1} .
$$

Hence, using the defining relations (2.11), we get

$$
\begin{array}{ll}
\left\{a_{i j}, a_{k l}\right\}=0 & \text { if } i>j>k>l \\
\left\{a_{i j}, a_{k l}\right\}=0 & \text { if } i>k>l>j \\
\left\{a_{i j}, a_{k l}\right\}=2\left(a_{i k} a_{j l}-a_{k j} a_{i l}\right) & \text { if } i>k>j>l \\
\left\{a_{i j}, a_{j l}\right\}=a_{i j} a_{j l}-2 a_{i l} & \text { if } i>j>l \\
\left\{a_{i j}, a_{i l}\right\}=a_{i j} a_{i l}-2 a_{l j} & \text { if } i>l>j \\
\left\{a_{i j}, a_{k j}\right\}=a_{i j} a_{k j}-2 a_{k i} & \text { if } \quad k>i>j .
\end{array}
$$

This coincides with the Poisson brackets of [2], 21], and [29], up to a constant factor if we interpret $a_{i j}$ as the $j i$-th entry of the upper triangular matrix.

We shall also use the presentation of the algebra $\mathrm{U}_{q}^{\prime}\left(\mathfrak{o}_{N}\right)$ due to Gavrilik and Klimyk [11. An isomorphism between the presentations was given by Noumi [24], a proof can be found in Iorgov and Klimyk [13]. Set $s_{i}=s_{i+1, i}$ for $i=1, \ldots, N-1$. Then the algebra $\mathrm{U}_{q}^{\prime}\left(\mathfrak{o}_{N}\right)$ is generated by the elements $s_{1}, \ldots, s_{N-1}$ subject only to the relations

$$
\begin{aligned}
s_{k} s_{k+1}^{2}-\left(q+q^{-1}\right) s_{k+1} s_{k} s_{k+1}+s_{k+1}^{2} s_{k} & =-q^{-1}\left(q-q^{-1}\right)^{2} s_{k}, \\
s_{k}^{2} s_{k+1}-\left(q+q^{-1}\right) s_{k} s_{k+1} s_{k}+s_{k+1} s_{k}^{2} & =-q^{-1}\left(q-q^{-1}\right)^{2} s_{k+1},
\end{aligned}
$$

for $k=1, \ldots, N-2$ (the Serre type relations), and

$$
s_{k} s_{l}=s_{l} s_{k}, \quad|k-l|>1 .
$$


It is easy to see that the subalgebra $\mathrm{U}_{q}^{\prime}\left(\mathfrak{o}_{N}\right) \subset \mathrm{U}_{q}\left(\mathfrak{g l}_{N}\right)$ is not preserved by the action of the braid group $B_{N}$ on $\mathrm{U}_{q}\left(\mathfrak{g l}_{N}\right)$ described above. Nevertheless, we have the following theorem.

Theorem 2.1. For $i=1, \ldots, N-1$ the assignment

$$
\begin{aligned}
\beta_{i}: s_{i+1} & \mapsto \frac{1}{q-q^{-1}}\left(q s_{i+1} s_{i}-s_{i} s_{i+1}\right) \\
s_{i-1} & \mapsto \frac{1}{q-q^{-1}}\left(s_{i} s_{i-1}-q s_{i-1} s_{i}\right) \\
s_{i} & \mapsto-s_{i} \quad \text { if } \quad k \neq i-1, i, i+1,
\end{aligned}
$$

defines an action of the braid group $B_{N}$ on $\mathrm{U}_{q}^{\prime}\left(\mathfrak{o}_{N}\right)$ by automorphisms.

Proof. We verify first that the images of the generators $s_{1}, \ldots, s_{N-1}$ under $\beta_{i}$ satisfy the defining relations of $\mathrm{U}_{q}^{\prime}\left(\mathfrak{o}_{N}\right)$. A nontrivial calculation is only required to verify that the images of the pairs of generators $\beta_{i}\left(s_{k}\right)$ and $\beta_{i}\left(s_{k+1}\right)$ with $k=i-2, i-1, i, i+1$ satisfy both Serre type relations, and that the images $\beta_{i}\left(s_{i-1}\right)$ and $\beta_{i}\left(s_{i+1}\right)$ commute. Observe that by (2.11), the image of $s_{i+1}$ can also be written as

$$
\beta_{i}: s_{i+1} \mapsto s_{i+2, i}
$$

Hence, for $k=i+1$ we need to verify that

$$
\begin{aligned}
s_{i+2, i} s_{i+3, i+2}^{2}-\left(q+q^{-1}\right) s_{i+3, i+2} s_{i+2, i} s_{i+3, i+2}+s_{i+3, i+2}^{2} s_{i+2, i} & \\
& =-q^{-1}\left(q-q^{-1}\right)^{2} s_{i+2, i} .
\end{aligned}
$$

We shall verify the following more general relation in $\mathrm{U}_{q}^{\prime}\left(\mathfrak{o}_{N}\right)$,

$$
s_{i j} s_{k i}^{2}-\left(q+q^{-1}\right) s_{k i} s_{i j} s_{k i}+s_{k i}^{2} s_{i j}=-q^{-1}\left(q-q^{-1}\right)^{2} s_{i j}
$$

where $k>i>j$. Indeed, the left hand side equals

$$
-\left(q s_{k i} s_{i j}-s_{i j} s_{k i}\right) s_{k i}+q^{-1} s_{k i}\left(q s_{k i} s_{i j}-s_{i j} s_{k i}\right) .
$$

However, by (2.11) we have

$$
q s_{k i} s_{i j}-s_{i j} s_{k i}=\left(q-q^{-1}\right) s_{k j}
$$

so that (2.16) becomes

$$
-q^{-1}\left(q-q^{-1}\right)\left(q s_{k j} s_{k i}-s_{k i} s_{k j}\right)
$$


which equals $-q^{-1}\left(q-q^{-1}\right)^{2} s_{i j}$ by (2.11) thus proving (2.15). The second Serre type relation for the images $\beta_{i}\left(s_{i+1}\right)$ and $\beta_{i}\left(s_{i+2}\right)$ follows from a more general relation in $\mathrm{U}_{q}^{\prime}\left(\mathfrak{o}_{N}\right)$,

$$
s_{i j}^{2} s_{k i}-\left(q+q^{-1}\right) s_{i j} s_{k i} s_{i j}+s_{k i} s_{i j}^{2}=-q^{-1}\left(q-q^{-1}\right)^{2} s_{k i},
$$

where $k>i>j$, and which is verified in the same way as (2.15). Next, the Serre type relations for the images $\beta_{i}\left(s_{i}\right)$ and $\beta_{i}\left(s_{i+1}\right)$ follow respectively from the relations

$$
s_{i j}^{2} s_{k j}-\left(q+q^{-1}\right) s_{i j} s_{k j} s_{i j}+s_{k j} s_{i j}^{2}=-q^{-1}\left(q-q^{-1}\right)^{2} s_{i j}
$$

and

$$
s_{i j}^{2} s_{k j}-\left(q+q^{-1}\right) s_{i j} s_{k j} s_{i j}+s_{k j} s_{i j}^{2}=-q^{-1}\left(q-q^{-1}\right)^{2} s_{k j},
$$

where $k>i>j$, which both are implied by (2.11). The Serre type relations for the pairs $\beta_{i}\left(s_{i-1}\right), \beta_{i}\left(s_{i}\right)$ and $\beta_{i}\left(s_{i-2}\right), \beta_{i}\left(s_{i-1}\right)$ can now be verified by using the involutive automorphism $\omega$ of $\mathrm{U}_{q}^{\prime}\left(\mathfrak{o}_{N}\right)$ which is defined on the generators by

$$
s_{k} \mapsto s_{N-k}, \quad k=1, \ldots, N-1
$$

We have

$$
\begin{aligned}
\omega: \beta_{i}\left(s_{i-2}\right) & \mapsto \beta_{N-i}\left(s_{N-i+2}\right), \\
\beta_{i}\left(s_{i-1}\right) & \mapsto-\beta_{N-i}\left(s_{N-i+1}\right), \\
\beta_{i}\left(s_{i}\right) & \mapsto \beta_{N-i}\left(s_{N-i}\right),
\end{aligned}
$$

and so the desired relations are implied by the Serre type relations for the pairs of the images $\beta_{j}\left(s_{j}\right), \beta_{j}\left(s_{j+1}\right)$ and $\beta_{j}\left(s_{j+1}\right), \beta_{j}\left(s_{j+2}\right)$ with $j=N-i$.

Now we verify that the images $\beta_{i}\left(s_{i-1}\right)$ and $\beta_{i}\left(s_{i+1}\right)$ commute, that is,

$$
\left(s_{i} s_{i-1}-q s_{i-1} s_{i}\right)\left(q s_{i+1} s_{i}-s_{i} s_{i+1}\right)=\left(q s_{i+1} s_{i}-s_{i} s_{i+1}\right)\left(s_{i} s_{i-1}-q s_{i-1} s_{i}\right) .
$$

By the Serre type relations we have

$$
s_{i}^{2} s_{i+1}-\left(q+q^{-1}\right) s_{i} s_{i+1} s_{i}+s_{i+1} s_{i}^{2}=-q^{-1}\left(q-q^{-1}\right)^{2} s_{i+1}
$$

and

$$
s_{i}^{2} s_{i-1}-\left(q+q^{-1}\right) s_{i} s_{i-1} s_{i}+s_{i-1} s_{i}^{2}=-q^{-1}\left(q-q^{-1}\right)^{2} s_{i-1} .
$$

Multiply the first of these relations by $s_{i-1}$ and the second by $s_{i+1}$ from the left. Taking the difference we come to

$$
s_{i-1} s_{i}^{2} s_{i+1}-\left(q+q^{-1}\right) s_{i-1} s_{i} s_{i+1} s_{i}=s_{i+1} s_{i}^{2} s_{i-1}-\left(q+q^{-1}\right) s_{i+1} s_{i} s_{i-1} s_{i} .
$$

Now repeat the same calculation but multiply the Serre type relations by $s_{i-1}$ and $s_{i+1}$, respectively, from the right. This gives

$$
s_{i-1} s_{i}^{2} s_{i+1}-\left(q+q^{-1}\right) s_{i} s_{i-1} s_{i} s_{i+1}=s_{i+1} s_{i}^{2} s_{i-1}-\left(q+q^{-1}\right) s_{i} s_{i+1} s_{i} s_{i-1} \text {. }
$$


Hence,

$$
s_{i-1} s_{i} s_{i+1} s_{i}-s_{i+1} s_{i} s_{i-1} s_{i}=s_{i} s_{i-1} s_{i} s_{i+1}-s_{i} s_{i+1} s_{i} s_{i-1}
$$

and (2.18) follows.

Thus, each $\beta_{i}$ with $i=1, \ldots, N-1$ defines a homomorphism $\mathrm{U}_{q}^{\prime}\left(\mathfrak{o}_{N}\right) \rightarrow \mathrm{U}_{q}^{\prime}\left(\mathfrak{o}_{N}\right)$. Now observe that $\beta_{i}$ is invertible with the inverse given by

$$
\begin{aligned}
\beta_{i}^{-1}: s_{i+1} & \mapsto \frac{1}{q-q^{-1}}\left(s_{i+1} s_{i}-q s_{i} s_{i+1}\right) \\
s_{i-1} & \mapsto \frac{1}{q-q^{-1}}\left(q s_{i} s_{i-1}-s_{i-1} s_{i}\right) \\
s_{i} & \mapsto-s_{i} \quad \text { if } \quad k \neq i-1, i, i+1,
\end{aligned}
$$

and so $\beta_{i}$ and $\beta_{i}^{-1}$ are mutually inverse automorphisms of $\mathrm{U}_{q}^{\prime}\left(\mathfrak{o}_{N}\right)$.

Finally, we verify that the automorphisms $\beta_{i}$ satisfy the braid group relations. It suffices to check that for each generator $s_{k}$ we have

$$
\beta_{i} \beta_{i+1} \beta_{i}\left(s_{k}\right)=\beta_{i+1} \beta_{i} \beta_{i+1}\left(s_{k}\right)
$$

for $i=1, \ldots, N-2$, and

$$
\beta_{i} \beta_{j}\left(s_{k}\right)=\beta_{j} \beta_{i}\left(s_{k}\right)
$$

for $|i-j|>1$. Clearly, the only nontrivial cases of (2.19) are $k=i-1, i, i+1, i+2$ while (2.20) is obvious for all cases except for $j=i+2$ and $k=i+1$. Take $k=i-1$ in (2.19). We have $\beta_{i+1}\left(s_{i-1}\right)=s_{i-1}$ while

$$
\beta_{i}: s_{i-1} \mapsto \frac{1}{q-q^{-1}}\left(s_{i} s_{i-1}-q s_{i-1} s_{i}\right)=q s_{i+1, i-1}-q s_{i+1, i} s_{i, i-1},
$$

where we have used (2.11). Furthermore, using again (2.11), we find

$$
\begin{aligned}
\beta_{i+1} \beta_{i}: s_{i-1} \mapsto q^{2} s_{i+2, i-1}-q^{2} s_{i+2, i+1} s_{i+1, i-1} & \\
& -q^{2} s_{i+2, i} s_{i, i-1}+q^{2} s_{i+2, i+1} s_{i+1, i} s_{i, i-1} .
\end{aligned}
$$

It remains to verify with the use of (2.11) that this element is stable under the action of $\beta_{i}$. The remaining cases of (2.19) and (2.20) are verified with similar and even simpler calculations.

Corollary 2.2. In terms of the generators $s_{k l}$ of the algebra $\mathrm{U}_{q}^{\prime}\left(\mathfrak{o}_{N}\right)$, for each index $i=1, \ldots, N-1$ the action of $\beta_{i}$ is given by

$$
\begin{aligned}
& \beta_{i}: s_{i+1, i} \mapsto-s_{i+1, i} \\
& s_{i k} \mapsto q s_{i+1, k}-q s_{i+1, i} s_{i k}, \quad s_{i+1, k} \mapsto s_{i k}, \quad \text { if } \quad k \leqslant i-1 \\
& s_{l i} \mapsto q^{-1} s_{l, i+1}-s_{l i} s_{i+1, i}, \quad s_{l, i+1} \mapsto s_{l i}, \quad \text { if } l \geqslant i+2 \\
& s_{k l} \mapsto s_{k l} \quad \text { in all remaining cases. }
\end{aligned}
$$


Proof. This follows from the defining relations (2.11). Indeed, the elements $s_{k l}$ can be expressed in terms of the generators $s_{1}, \ldots, s_{N-1}$ by induction, using the relations

$$
s_{k l}=\frac{1}{q-q^{-1}}\left(q s_{k j} s_{j l}-s_{j l} s_{k j}\right), \quad k>j>l .
$$

This determines the action of $\beta_{i}$ on the elements $s_{k l}$ and the formulas are verified by induction.

Remark 2.3. It is possible to prove that the formulas of Corollary 2.2 define an action of the braid group $B_{N}$ on $\mathrm{U}_{q}^{\prime}\left(\mathfrak{o}_{N}\right)$ by automorphisms only using the presentation (2.11). However, this leads to a slightly longer calculations as compared with the proof of Theorem 2.1.

Note also that the universal enveloping algebra $\mathrm{U}\left(\mathfrak{o}_{N}\right)$ can be obtained as a specialization of $\mathrm{U}_{q}^{\prime}\left(\mathfrak{o}_{N}\right)$ in the limit $q \rightarrow 1$; see [19] for a precise formulation. In this limit the elements $s_{i j} /\left(q-q^{-1}\right)$ with $i>j$ specialize to the generators $F_{i j}$ of $\mathfrak{o}_{N}$, where $F_{i j}=E_{i j}-E_{j i}$. Hence the action of $B_{N}$ on $\mathrm{U}_{q}^{\prime}\left(\mathfrak{o}_{N}\right)$ specializes to the action of the symmetric group $\mathfrak{S}_{N}$ on $\mathrm{U}\left(\mathfrak{o}_{N}\right)$ by permutations of the indices of the $F_{i j}$.

The mapping (2.17) can also be extended to the entire algebra $\mathrm{U}_{q}^{\prime}\left(\mathfrak{o}_{N}\right)$ as an antiautomorphism. This is readily verified with the use of the Serre type relations. We denote this involutive anti-automorphism of $\mathrm{U}_{q}^{\prime}\left(\mathfrak{o}_{N}\right)$ by $\omega^{\prime}$.

Proposition 2.4. The action of $\omega^{\prime}$ on the generators $s_{k l}$ is given by

$$
\omega^{\prime}: s_{k l} \mapsto s_{N-l+1, N-k+1}, \quad 1 \leqslant l<k \leqslant N .
$$

Moreover, we have the relations

$$
\omega^{\prime} \beta_{i} \omega^{\prime}=\beta_{N-i}^{-1}, \quad i=1, \ldots, N-1
$$

where the automorphisms $\beta_{i}$ of $\mathrm{U}_{q}^{\prime}\left(\mathfrak{o}_{N}\right)$ are defined in Theorem 2.1.

Proof. The defining relations (2.11) imply that the mapping (2.22) defines an antiautomorphism of $\mathrm{U}_{q}^{\prime}\left(\mathfrak{o}_{N}\right)$. Obviously, the images of the generators $s_{k}$ are found by (2.17). The second part of the proposition is verified by comparing the images of the generators $s_{k}$ under the automorphisms on both sides of (2.23).

Observe that the image of the matrix $S$ under $\omega^{\prime}$ is given by $\omega^{\prime}: S \mapsto S^{\prime}$, where the prime denotes the transposition with respect to the second diagonal.

Now consider the involutive automorphism $\omega$ of $\mathrm{U}_{q}^{\prime}\left(\mathfrak{o}_{N}\right)$ defined by the mapping (2.17). 
Proposition 2.5. The image of the matrix $S$ under $\omega$ is given by

$$
\omega: S \mapsto\left(1-q^{-1}\right) I+q^{-1} D\left(S^{-1}\right)^{\prime} D^{-1}
$$

where $I$ is the identity matrix and $D=\operatorname{diag}\left(-q,(-q)^{2}, \ldots,(-q)^{N}\right)$. In terms of the generators, this can be written as

$$
\omega: s_{k l} \mapsto(-q)^{k-l-1} \sum_{N-l+1>r_{1}>\cdots>r_{p}>N-k+1}(-1)^{p} s_{N-l+1, r_{1}} s_{r_{1} r_{2}} \ldots s_{r_{p}, N-k+1}, \quad k>l,
$$

summed over $p \geqslant 0$ and the indices $r_{1}, \ldots, r_{p}$.

Proof. The elements $s_{k l}$ can be expressed in terms of the generators $s_{1}, \ldots, s_{N-1}$ by (2.21). The formula for $\omega\left(s_{k l}\right)$ is then verified by induction on $k-l$. The matrix form (2.24) is implied by the relation

$$
\left(S^{-1}\right)_{k l}=\sum_{k>r_{1}>\cdots>r_{p}>l}(-1)^{p+1} s_{k, r_{1}} s_{r_{1} r_{2}} \ldots s_{r_{p}, l}, \quad k>l,
$$

summed over $p \geqslant 0$ and the indices $r_{1}, \ldots, r_{p}$.

For any diagonal matrix $C=\operatorname{diag}\left(c_{1}, \ldots, c_{N}\right)$ the relation (2.8) is preserved by the transformation $S \mapsto C S C$. Indeed, the entries of $S$ are then transformed as $s_{i j} \mapsto s_{i j} c_{i} c_{j}$ and the claim is immediate from (2.10). This implies that if $c_{i}^{2}=1$ for all $i$ then the mapping $\varsigma: S \mapsto C S C$ defines an automorphism of $\mathrm{U}_{q}^{\prime}\left(\mathfrak{o}_{N}\right)$. Therefore, Propositions 2.4 and 2.5 imply the following corollary.

Corollary 2.6. The mapping

$$
\rho: S \mapsto\left(1-q^{-1}\right) I+q^{-1} H S^{-1} H^{-1}
$$

where $H=\operatorname{diag}\left(q, q^{2}, \ldots, q^{N}\right)$, defines an involutive anti-automorphism of $\mathrm{U}_{q}^{\prime}\left(\mathfrak{o}_{N}\right)$.

Proof. We obviously have $\rho=\varsigma \circ \omega^{\prime} \circ \omega$ for an appropriate automorphism $\varsigma$. Hence $\rho$ is an anti-automorphism. We have

$$
\rho: s_{k} \mapsto-s_{k}, \quad k=1, \ldots, N-1
$$

and so $\rho$ is involutive.

We can now recover the braid group action on the algebra $\mathcal{P}_{N}$; see Dubrovin [8], Bondal [2]. 
Corollary 2.7. The braid group $B_{N}$ acts on the algebra $\mathcal{P}_{N}$ by

$$
\begin{aligned}
\beta_{i}: & a_{i+1, i} & & \\
& a_{i k} \mapsto a_{i+1, k}-a_{i+1, i} a_{i k}, & & \\
& a_{l i+1, k} \mapsto a_{l, i+1}-a_{l i} a_{i+1, i}, & & \text { if } k \leqslant i-1 \\
& a_{k l} \mapsto a_{k l} & & \text { if } l \geqslant i+2 \\
& & \text { in all remaining cases, }, &
\end{aligned}
$$

where $i=1, \ldots, N-1$. Moreover, the Poisson bracket on $\mathcal{P}_{N}$ in invariant under this action.

Proof. This is immediate from Corollary 2.2.

We combine the variables $a_{i j}$ into the lower triangular matrix $A=\left[a_{i j}\right]$ where we set $a_{i i}=1$ for all $i$ and $a_{i j}=0$ for $i<j$.

Corollary 2.8. The mappings

$$
\varrho: A \mapsto A^{-1} \quad \text { and } \quad \varpi: A \mapsto A^{\prime}
$$

define anti-automorphisms of the Poisson bracket on $\mathcal{P}_{N}$. Explicitly, the image of $a_{k l}$ under $\varrho$ is given by

$$
\varrho: a_{k l} \mapsto \sum_{k>r_{1}>\cdots>r_{p}>l}(-1)^{p+1} a_{k r_{1}} a_{r_{1} r_{2}} \ldots a_{r_{p}, l}, \quad k>l
$$

summed over $p \geqslant 0$ and the indices $r_{1}, \ldots, r_{p}$.

Proof. This follows from Proposition 2.4 and Corollary 2.6 by taking $q=1$.

\section{Casimir elements of the Poisson algebra $\mathcal{P}_{N}$}

Using the relationship between the twisted quantized enveloping algebra $\mathrm{U}_{q}^{\prime}\left(\mathfrak{o}_{N}\right)$ and the Poisson algebra $\mathcal{P}_{N}$, we can get families of invariants of $\mathcal{P}_{N}$ by taking the classical limit $q \rightarrow 1$ in the constructions of [19], [12] and [25]. First, we recall the construction of Casimir elements for the algebra $\mathrm{U}_{q}^{\prime}\left(\mathfrak{o}_{N}\right)$ given in [19]. Consider the $q$-permutation operator $P^{q} \in$ End $\left(\mathbb{C}^{N} \otimes \mathbb{C}^{N}\right)$ defined by

$$
P^{q}=\sum_{i} E_{i i} \otimes E_{i i}+q \sum_{i>j} E_{i j} \otimes E_{j i}+q^{-1} \sum_{i<j} E_{i j} \otimes E_{j i}
$$

Introduce the multiple tensor product $\mathrm{U}_{q}^{\prime}\left(\mathfrak{o}_{N}\right) \otimes\left(\text { End } \mathbb{C}^{N}\right)^{\otimes r}$. The action of the symmetric group $\mathfrak{S}_{r}$ on the space $\left(\mathbb{C}^{N}\right)^{\otimes r}$ can be defined by setting $\sigma_{i} \mapsto P_{\sigma_{i}}^{q}:=P_{i, i+1}^{q}$ 
for $i=1, \ldots, r-1$, where $\sigma_{i}$ denotes the transposition $(i, i+1)$. If $\sigma=\sigma_{i_{1}} \cdots \sigma_{i_{l}}$ is a reduced decomposition of an element $\sigma \in \mathfrak{S}_{r}$ we set $P_{\sigma}^{q}=P_{\sigma_{i_{1}}}^{q} \cdots P_{\sigma_{i_{l}}}^{q}$. We denote by $A_{r}^{q}$ the $q$-antisymmetrizer

$$
A_{r}^{q}=\sum_{\sigma \in \mathfrak{S}_{r}} \operatorname{sgn} \sigma \cdot P_{\sigma}^{q}
$$

Now take $r=N$. We have the relation

$$
\begin{aligned}
A_{N}^{q} S_{1}(u) R_{12}^{t} \cdots R_{1 N}^{t} S_{2}\left(u q^{-2}\right) & R_{23}^{t} \cdots R_{2 N}^{t} S_{3}\left(u q^{-4}\right) \\
& \times \cdots R_{N-1, N}^{t} S_{N}\left(u q^{-2 N+2}\right) \\
=S_{N}\left(u q^{-2 N+2}\right) R_{N-1, N}^{t} \cdots S_{3}\left(u q^{-4}\right) & R_{2 N}^{t} \cdots R_{23}^{t} S_{2}\left(u q^{-2}\right) \\
& \times R_{1 N}^{t} \cdots R_{12}^{t} S_{1}(u) A_{N}^{q},
\end{aligned}
$$

where the following notation was used. The matrix $S(u)$ is defined by

$$
S(u)=S+q^{-1} u^{-1} \bar{S}
$$

where $u$ is a formal variable and $\bar{S}$ is the upper triangular matrix with ones on the diagonal whose $i j$-th entry is $\bar{s}_{i j}=q s_{j i}$ for $i<j$. Furthermore,

$$
R_{i j}^{t}=R_{i j}^{t}\left(u^{-1} q^{2 i-2}, u q^{-2 j+2}\right)
$$

with

$$
\begin{aligned}
R^{t}(u, v) & =(u-v) \sum_{i \neq j} E_{i i} \otimes E_{j j}+\left(q^{-1} u-q v\right) \sum_{i} E_{i i} \otimes E_{i i} \\
& +\left(q^{-1}-q\right) u \sum_{i>j} E_{j i} \otimes E_{j i}+\left(q^{-1}-q\right) v \sum_{i<j} E_{j i} \otimes E_{j i} .
\end{aligned}
$$

The subscripts in (3.3) indicate the copies of End $\mathbb{C}^{N}$ in $\mathrm{U}_{q}^{\prime}\left(\mathfrak{o}_{N}\right) \otimes\left(\text { End } \mathbb{C}^{N}\right)^{\otimes N}$ which are labelled by $1, \ldots, N$; cf. (2.1). The element (3.3) equals $A_{N}^{q}$ sdet $S(u)$, where sdet $S(u)$ is a rational function in $u$ (the Sklyanin determiant) valued in the center of $\mathrm{U}_{q}^{\prime}\left(\mathfrak{o}_{N}\right)$; see [19, Theorem 3.8 and Corollary 4.3].

Recall that the Poisson algebra $\mathcal{P}_{N}$ is the algebra of polynomials in the variables $a_{i j}$ with $i>j$ which are combined into the matrix $A=\left[a_{i j}\right]$ with $a_{i i}=1$ for all $i$ and $a_{i j}=0$ for $i<j$. The following theorem was proved in different ways by Nelson and Regge [22] and Bondal [2].

Theorem 3.1. The coefficients of the polynomial

$$
\operatorname{det}\left(A+\lambda A^{t}\right)=f_{0}+f_{1} \lambda+\cdots+f_{N} \lambda^{N}
$$

are Casimir elements of the Poisson algebra $\mathcal{P}_{N}$. 
Proof. We use the centrality of the Sklyanin determinant sdet $S(u)$ in $\mathrm{U}_{q}^{\prime}\left(\mathfrak{o}_{N}\right)$. Note that at $q=1$ the $q$-antisymmetrizer $A_{N}^{q}$ becomes the antisymmetrizer in $\left(\mathbb{C}^{N}\right)^{\otimes N}$, the element $R^{t}(u-v)$ becomes $u-v$ times the identity. Since the images of the elements $s_{i j}$ in $\mathcal{P}_{N}$ coincide with $a_{i j}$, the image of the matrix $S(u)$ is $A+u^{-1} A^{t}$. Hence, at $q=1$ the Sklyanin determinant sdet $S(u)$ becomes $\gamma(u) \operatorname{det}\left(A+u^{-1} A^{t}\right)$, where

$$
\gamma(u)=\left(u^{-1}-u\right)^{N(N-1) / 2} .
$$

Therefore, replacing $u$ with $\lambda^{-1}$ we thus prove that all coefficients of $\operatorname{det}\left(A+\lambda A^{t}\right)$ are Casimir elements for the Poisson bracket on $\mathcal{P}_{N}$.

Note that, as was proved in [2] and [22], the polynomial $\operatorname{det}\left(A+\lambda A^{t}\right)$ is invariant under the action of the braid group $B_{N}$.

Now we recall the construction of Casimir elements given in [12]. For all $i>j$ define the elements $s_{i j}^{+}$of $\mathrm{U}_{q}^{\prime}\left(\mathfrak{o}_{N}\right)$ by induction from the formulas

$$
s_{i j}^{+}=\frac{1}{q-q^{-1}}\left(s_{i, j+1}^{+} s_{j+1, j}-q s_{j+1, j} s_{i, j+1}^{+}\right), \quad i>j+1,
$$

and $s_{j+1, j}^{+}=s_{j+1, j}$ for $j=1, \ldots, N-1$. A straightforward calculation shows that these elements can be equivalently defined by

$$
s_{i j}^{+}=-q^{i-j-1}\left(S^{-1}\right)_{i j}, \quad i>j,
$$

where the entries of the inverse matrix are found from (2.25). Let $k$ be a positive integer such that $2 k \leqslant N$. For any subset $I=\left\{i_{1}<i_{2}<\cdots<i_{2 k}\right\}$ of $\{1, \ldots, N\}$ introduce the elements $\Phi_{I}$ and $\Phi_{I}^{+}$of $\mathrm{U}_{q}^{\prime}\left(\mathfrak{o}_{N}\right)$ by

$$
\Phi_{I}=\sum_{\sigma \in \mathfrak{S}_{2 k}}(-q)^{-\ell(\sigma)} s_{i_{\sigma(2)} i_{\sigma(1)}} \ldots s_{i_{\sigma(2 k)} i_{\sigma(2 k-1)}}
$$

and

$$
\Phi_{I}^{+}=\sum_{\sigma \in \mathfrak{S}_{2 k}}(-q)^{\ell(\sigma)} s_{i_{\sigma(2)} i_{\sigma(1)}}^{+} \ldots s_{i_{\sigma(2 k)} i_{\sigma(2 k-1)}}^{+},
$$

where $\ell(\sigma)$ is the length of the permutation $\sigma$, and the sums are taken over those permutations $\sigma \in \mathfrak{S}_{2 k}$ which satisfy the conditions

$$
i_{\sigma(2)}>i_{\sigma(1)}, \quad \ldots, \quad i_{\sigma(2 k)}>i_{\sigma(2 k-1)} \quad \text { and } \quad i_{\sigma(2)}<i_{\sigma(4)}<\cdots<i_{\sigma(2 k)} .
$$

Then according to [12], for each $k$ the element

$$
\phi_{k}=\sum_{I,|I|=2 k} q^{i_{1}+i_{2}+\cdots+i_{2 k}} \Phi_{I}^{+} \Phi_{I}
$$

belongs to the center of $\mathrm{U}_{q}^{\prime}\left(\mathfrak{o}_{N}\right)$. Moreover, in the case $N=2 n$ both elements $\Phi_{I_{0}}$ and $\Phi_{I_{0}}^{+}$with $I_{0}=\{1, \ldots, 2 n\}$ are also central. 
Remark 3.2. Our notation is related to [12] by

$$
s_{i j}=-q^{-1 / 2}\left(q-q^{-1}\right) I_{i j}^{-}, \quad s_{i j}^{+}=-q^{-1 / 2}\left(q-q^{-1}\right) I_{i j}^{+}, \quad i>j .
$$

Note also that the elements $\phi_{k}$ are $q$-analogues of the Casimir elements for the orthogonal Lie algebra $\mathfrak{o}_{N}$ constructed in [18]; see also [15].

Now return to the Poisson algebra $\mathcal{P}_{N}$. Recall that the Pfaffian of a $2 k \times 2 k$ skew symmetric matrix $H$ is given by

$$
\operatorname{Pf} H=\frac{1}{2^{k} k !} \sum_{\sigma \in \mathfrak{S}_{2 k}} \operatorname{sgn} \sigma \cdot H_{\sigma(1), \sigma(2)} \ldots H_{\sigma(2 k-1), \sigma(2 k)} .
$$

Given a lower triangular $N \times N$ matrix $B$ and a $2 k$-element subset $I$ of $\{1, \ldots, N\}$ as above, we denote by $\operatorname{Pf}_{I}(B)$ the Pfaffian of the $2 k \times 2 k$ submatrix $\left(B^{t}-B\right)_{I}$ of $B^{t}-B$ whose rows and columns are determined by the elements of $I$.

Theorem 3.3. For each positive integer $k$ such that $2 k \leqslant N$ the element

$$
c_{k}=(-1)^{k} \sum_{I,|I|=2 k} \operatorname{Pf}_{I}(A) \operatorname{Pf}_{I}\left(A^{-1}\right)
$$

is a Casimir element of $\mathcal{P}_{N}$. Moreover, in the case $N=2 n$ both $\operatorname{Pf}_{I_{0}}(A)$ and $\operatorname{Pf}_{I_{0}}\left(A^{-1}\right)$ with $I_{0}=\{1, \ldots, 2 n\}$ are also Casimir elements.

Proof. Observe that in the limit $q \rightarrow 1$ the elements $\Phi_{I}$ and $\Phi_{I}^{+}$specialize respectively to the Pfaffians

$$
\Phi_{I} \rightarrow \operatorname{Pf}_{I}(A), \quad \Phi_{I}^{+} \rightarrow(-1)^{k} \operatorname{Pf}_{I}\left(A^{-1}\right)
$$

Hence, the central element $\phi_{k}$ specializes to $c_{k}$.

Example 3.4. As the matrix elements of the inverse matrix $A^{-1}$ are found by the formula of Corollary 2.8, we have the following explicit formula for $c_{1}$,

$$
c_{1}=\sum_{i>r_{1}>\cdots>r_{p}>j}(-1)^{p} a_{i j} a_{i r_{1}} a_{r_{1} r_{2}} \ldots a_{r_{p} j}
$$

For $N=3$ it gives the Markov polynomial.

Corollary 3.5. The algebra of Casimir elements of $\mathcal{P}_{N}$ is generated by $c_{1}, \ldots, c_{n}$ for $N=2 n+1$, and by $c_{1}, \ldots, c_{n-1}, \operatorname{Pf}_{I_{0}}(A)$ if $N=2 n$. In both cases, the families of generators are algebraically independent. Moreover, $\operatorname{Pf}_{I_{0}}\left(A^{-1}\right)=(-1)^{n} \operatorname{Pf}_{I_{0}}(A)$. 
Proof. Since

$$
\operatorname{det}\left(A+\lambda A^{t}\right)=\lambda^{N} \operatorname{det}\left(A+\lambda^{-1} A^{t}\right),
$$

we have the relations $f_{N-i}=f_{i}$. Moreover, $f_{0}=f_{N}=1$ since $\operatorname{det} A=1$. It was proved in [2] that if $N=2 n+1$ is odd then the coefficients $f_{1}, \ldots, f_{n}$ are algebraically independent generators of the algebra of Casimir elements of $\mathcal{P}_{N}$. If $N=2 n$ is even then

$$
\operatorname{det}\left(A-A^{t}\right)=\operatorname{Pf}_{I_{0}}(A)^{2} .
$$

In this case, a family of algebraically independent generators of the algebra of Casimir elements of $\mathcal{P}_{N}$ is obtained by replacing any one of the elements $f_{1}, \ldots, f_{n}$ with $\operatorname{Pf}_{I_{0}}(A)$. The claim will be implied by the following identity

$$
\operatorname{det}\left(A+\lambda A^{t}\right)=\sum_{k=0}^{n}(-\lambda)^{k}(1+\lambda)^{N-2 k} c_{k} .
$$

Indeed, by the identity, the elements $f_{1}, \ldots, f_{n}$ can be expressed as linear combinations of $c_{1}, \ldots, c_{n}$. In order to verity (3.8), we use the observation of [2] that the Casimir elements of $\mathcal{P}_{N}$ are determined by their restrictions on a certain subspace $\mathcal{H}$ of matrices. If $N=2 n$ then $\mathcal{H}$ consists of the matrices of the form

$$
\left(\begin{array}{ll}
I & O \\
D & I
\end{array}\right)
$$

where $I$ and $O$ are the identity and zero $n \times n$ matrices, respectively, while $D=$ $\operatorname{diag}\left(d_{1}, \ldots, d_{n}\right)$ is an arbitrary diagonal matrix. If $N=2 n+1$ then $\mathcal{H}$ consists of the matrices obtained from (3.9) by inserting an extra row and column in the middle of the matrix whose only nonzero entry is 1 at their intersection. So, by Theorems 3.1 and 3.3, we only need to verify (3.8) for the matrices $A \in \mathcal{H}$. However, in this case the element $c_{k}$ coincides with the elementary symmetric polynomial

$$
c_{k}=\sum_{r_{1}<\cdots<r_{k}} d_{r_{1}}^{2} \ldots d_{r_{k}}^{2},
$$

while

$$
\operatorname{det}\left(A+\lambda A^{t}\right)=\prod_{i=1}^{n}\left((1+\lambda)^{2}-\lambda d_{i}^{2}\right)
$$

if $N=2 n$, and

$$
\operatorname{det}\left(A+\lambda A^{t}\right)=(1+\lambda) \prod_{i=1}^{n}\left((1+\lambda)^{2}-\lambda d_{i}^{2}\right)
$$

if $N=2 n+1$. This gives (3.8). To verify the last statement of the corollary, put $\lambda=-1$ into (3.8) with $N=2 n$. Together with (3.7) this gives $c_{n}=\operatorname{Pf}_{I_{0}}(A)^{2}$, so that the statement follows from (3.6) with $k=n$. 
Finally, we consider the invariants of the Poisson bracket on $\mathcal{P}_{N}$ which can obtained from the construction of the Casimir elements of $\mathrm{U}_{q}^{\prime}\left(\mathfrak{o}_{N}\right)$ given in [25].

Theorem 3.6. The elements

$$
\operatorname{tr}\left(A^{-1} A^{t}\right)^{k}, \quad k=1,2, \ldots,
$$

are Casimir elements of $\mathcal{P}_{N}$.

Proof. This follows by taking the classical limit of the Casimir elements of [25]. Alternatively, this is also implied by Theorem 3.1 and the Liouville formula

$$
\sum_{k=1}^{\infty}(-1)^{k-1} \lambda^{k-1} \operatorname{tr} H^{k}=\frac{d}{d \lambda} \ln \operatorname{det}(1+\lambda H)
$$

which holds for any square matrix $H$. We apply it to the matrix $H=A^{-1} A^{t}$ and observe that $\operatorname{det}\left(A+\lambda A^{t}\right)=\operatorname{det}(1+\lambda H)$ since $\operatorname{det} A=1$.

\section{A new Poisson algebra}

Here we use the symplectic version of the twisted quantized enveloping algebra introduced by Noumi [24] to define a new Poisson algebra and calculate its Casimir elements.

The twisted quantized enveloping algebra $\mathrm{U}_{q}^{\prime}\left(\mathfrak{s p}_{2 n}\right)$ is an associative algebra generated by elements $s_{i j}, i, j \in\{1, \ldots, 2 n\}$ and $s_{i, i+1}^{-1}, i=1,3, \ldots, 2 n-1$. The generators $s_{i j}$ are zero for $j=i+1$ with even $i$, and for $j \geqslant i+2$ and all $i$. We combine the $s_{i j}$ into a matrix $S$ as in (2.2),

$$
S=\sum_{i, j} s_{i j} \otimes E_{i j}
$$

so that $S$ has a block-triangular form with $n$ diagonal $2 \times 2$-blocks,

$$
S=\left(\begin{array}{ccccccc}
s_{11} & s_{12} & 0 & 0 & \cdots & 0 & 0 \\
s_{21} & s_{22} & 0 & 0 & \cdots & 0 & 0 \\
s_{31} & s_{32} & s_{33} & s_{34} & \cdots & 0 & 0 \\
s_{41} & s_{42} & s_{43} & s_{44} & \cdots & 0 & 0 \\
\vdots & \vdots & \vdots & \vdots & \ddots & \vdots & \vdots \\
s_{2 n-1,1} & s_{2 n-1,2} & s_{2 n-1,3} & s_{2 n-1,4} & \cdots & s_{2 n-1,2 n-1} & s_{2 n-1,2 n} \\
s_{2 n, 1} & s_{2 n, 2} & s_{2 n, 3} & s_{2 n, 4} & \cdots & s_{2 n, 2 n-1} & s_{2 n, 2 n}
\end{array}\right)
$$

The defining relations of $\mathrm{U}_{q}^{\prime}\left(\mathfrak{s p}_{2 n}\right)$ have the form of a reflection equation (2.8) together with

$$
s_{i, i+1} s_{i, i+1}^{-1}=s_{i, i+1}^{-1} s_{i, i+1}=1
$$


and

$$
s_{i+1, i+1} s_{i i}-q^{2} s_{i+1, i} s_{i, i+1}=q^{3}
$$

for $i=1,3, \ldots, 2 n-1$. More explicitly, the relations (2.8) have exactly the same form (2.10) as in the orthogonal case.

Recall the quantized enveloping algebra $\mathrm{U}_{q}\left(\mathfrak{g l}_{2 n}\right)$ defined in Section 2, Introduce the block-diagonal $2 n \times 2 n$ matrix $G$ by

$$
G=\left(\begin{array}{ccccc}
0 & q & \cdots & 0 & 0 \\
-1 & 0 & \cdots & 0 & 0 \\
\vdots & \vdots & \ddots & \vdots & \vdots \\
0 & 0 & \cdots & 0 & q \\
0 & 0 & \cdots & -1 & 0
\end{array}\right)
$$

We can regard $\mathrm{U}_{q}^{\prime}\left(\mathfrak{s p}_{2 n}\right)$ as a subalgebra of $\mathrm{U}_{q}\left(\mathfrak{g l}_{2 n}\right)$ by setting $S=T G \bar{T}^{t}$, or in terms of generators,

$$
s_{i j}=q \sum_{k=1}^{n} t_{i, 2 k-1} \bar{t}_{j, 2 k}-\sum_{k=1}^{n} t_{i, 2 k} \bar{t}_{j, 2 k-1}
$$

see [24] and [19] for the proofs.

Define the extended twisted quantized enveloping algebra $\hat{\mathrm{U}}_{q}^{\prime}\left(\mathfrak{s p}_{2 n}\right)$ as follows. This is an associative algebra generated by elements $s_{i j}, i, j \in\{1, \ldots, 2 n\}$ where $s_{i j}=0$ for $j=i+1$ with even $i$, and for $j \geqslant i+2$ and all $i$. The defining relations are given by (2.8) or, equivalently, by (2.10). We use the same symbols as for the generators of $\mathrm{U}_{q}^{\prime}\left(\mathfrak{s p}_{2 n}\right)$; a confusion should be avoided as we indicate which algebra is considered at any moment. This definition essentially coincides with the original one due to Noumi [24]. Note that, in comparison with $\mathrm{U}_{q}^{\prime}\left(\mathfrak{s p}_{2 n}\right)$, we neither require the elements $s_{i, i+1}$ with odd $i$ to be invertible, nor we impose the relations (4.3).

An analogue of the Poincaré-Birkhoff-Witt theorem for the algebra $\hat{U}_{q}^{\prime}\left(\mathfrak{s p}_{2 n}\right)$ follows from [17, Corollary 3.4]. As with the algebra $\mathrm{U}_{q}^{\prime}\left(\mathfrak{o}_{N}\right)$, this theorem implies that at $q=1$ the extended twisted quantized enveloping algebra $\hat{\mathrm{U}}_{q}^{\prime}\left(\mathfrak{s p}_{2 n}\right)$ specializes to the algebra $\hat{\mathcal{P}}_{2 n}$ of polynomials in $2 n^{2}+2 n$ variables. We denote the variables by $a_{i j}$ with the same restrictions on the indices $i, j$ as for the elements $s_{i j}$, so that $s_{i j}$ specializes to $a_{i j}$. We shall combine the variables $a_{i j}$ into a matrix $A$ which has a 
block-triangular form with $n$ diagonal $2 \times 2$-blocks,

$$
A=\left(\begin{array}{ccccccc}
a_{11} & a_{12} & 0 & 0 & \cdots & 0 & 0 \\
a_{21} & a_{22} & 0 & 0 & \cdots & 0 & 0 \\
a_{31} & a_{32} & a_{33} & a_{34} & \cdots & 0 & 0 \\
a_{41} & a_{42} & a_{43} & a_{44} & \cdots & 0 & 0 \\
\vdots & \vdots & \vdots & \vdots & \ddots & \vdots & \vdots \\
a_{2 n-1,1} & a_{2 n-1,2} & a_{2 n-1,3} & a_{2 n-1,4} & \cdots & a_{2 n-1,2 n-1} & a_{2 n-1,2 n} \\
a_{2 n, 1} & a_{2 n, 2} & a_{2 n, 3} & a_{2 n, 4} & \cdots & a_{2 n, 2 n-1} & a_{2 n, 2 n}
\end{array}\right)
$$

Theorem 4.1. The algebra $\hat{\mathcal{P}}_{2 n}$ possesses the Poisson bracket defined by

$$
\begin{aligned}
\left\{a_{i j}, a_{k l}\right\} & =\left(\delta_{i k}+\delta_{j k}-\delta_{i l}-\delta_{j l}\right) a_{i j} a_{k l} \\
& -2\left(\delta_{l<j}-\delta_{i<k}\right) a_{k j} a_{i l}-2 \delta_{l<i} a_{k i} a_{l j}+2 \delta_{j<k} a_{i k} a_{j l} .
\end{aligned}
$$

Proof. We define the Poisson bracket on $\hat{\mathcal{P}}_{2 n}$ by the same rule (2.13) as in the orthogonal case. The explicit formulas for the values $\left\{a_{i j}, a_{k l}\right\}$ follow from (2.10).

Remark 4.2. Both in the orthogonal and symplectic case, the Poisson brackets of $\mathcal{P}=\mathcal{P}_{N}$ or $\mathcal{P}=\hat{\mathcal{P}}_{2 n}$ can be written in a uniform way in a matrix form. Introducing the elements of $\mathcal{P} \otimes$ End $\mathbb{C}^{N} \otimes$ End $\mathbb{C}^{N}$ by

$$
A_{1}=\sum_{i, j} a_{i j} \otimes E_{i j} \otimes 1, \quad A_{2}=\sum_{i, j} a_{i j} \otimes 1 \otimes E_{i j},
$$

we have

$$
\left\{A_{1}, A_{2}\right\}=\left[r, A_{1} A_{2}\right]+A_{1} r^{t} A_{2}-A_{2} r^{t} A_{1}
$$

where

$$
r=\sum_{i} E_{i i} \otimes E_{i i}+2 \sum_{i<j} E_{i j} \otimes E_{j i}, \quad r^{t}=\sum_{i} E_{i i} \otimes E_{i i}+2 \sum_{i<j} E_{j i} \otimes E_{j i} .
$$

This follows from (2.8) and the observation that

$$
r=\left.\frac{R-I \otimes I}{q-1}\right|_{q=1} .
$$

Theorem 4.3. The elements

$$
a_{i+1, i+1} a_{i i}-a_{i+1, i} a_{i, i+1}, \quad i=1,3, \ldots, 2 n-1,
$$

and the coefficients of the polynomial

$$
\operatorname{det}\left(A+\lambda A^{t}\right)=f_{0}+f_{1} \lambda+\cdots+f_{2 n} \lambda^{2 n}
$$

are Casimir elements of the Poisson algebra $\hat{\mathcal{P}}_{2 n}$. 
Proof. For any $i=1,3, \ldots, 2 n-1$ the element

$$
s_{i+1, i+1} s_{i i}-q^{2} s_{i+1, i} s_{i, i+1}
$$

belongs to the center of the algebra $\hat{\mathrm{U}}_{q}^{\prime}\left(\mathfrak{s p}_{2 n}\right)$; see [19, Section 2.2]. This implies the claim for the elements (4.5).

We proceed as in the proof of Theorem 3.1. The relation (3.3) holds in the same form with the matrix $S(u)$ now given by

$$
S(u)=S+q u^{-1} \bar{S}
$$

where the matrix elements $\bar{s}_{i j}$ of $\bar{S}$ are defined as follows. For any $i=1,3, \ldots, 2 n-1$ we have

$$
\begin{aligned}
\bar{s}_{i i} & =-q^{-2} s_{i i}, & \bar{s}_{i+1, i+1} & =-q^{-2} s_{i+1, i+1}, \\
\bar{s}_{i+1, i} & =-q^{-1} s_{i, i+1}, & \bar{s}_{i, i+1} & =-q^{-1} s_{i+1, i}+\left(1-q^{-2}\right) s_{i, i+1},
\end{aligned}
$$

while

$$
\bar{s}_{k l}=-q^{-1} s_{l k}
$$

for $k<l$ except for the pairs $k=i, l=i+1$, with odd $i$, and the remaining entries of $\bar{S}$ are equal to zero. The element (3.3) equals $A_{N}^{q}$ sdet $S(u)$, where sdet $S(u)$ is the Sklyanin determiant of the matrix $S(u)$. This is a rational function in $u$ valued in the (extended) twisted quantized enveloping algebra. When the values are considered in the algebra $\mathrm{U}_{q}^{\prime}\left(\mathfrak{s p}_{2 n}\right)$, they are contained in the center of $\mathrm{U}_{q}^{\prime}\left(\mathfrak{s p}_{2 n}\right)$, as proved in [19, Theorem 3.15 and Corollary 4.3]. The same property holds for the algebra $\hat{U}_{q}^{\prime}\left(\mathfrak{s p}_{2 n}\right)$, that is, when the values of the function sdet $S(u)$ are regarded as elements of the extended algebra $\hat{\mathrm{U}}_{q}^{\prime}\left(\mathfrak{s p}_{2 n}\right)$, they belong to the center of $\hat{\mathrm{U}}_{q}^{\prime}\left(\mathfrak{s p}_{2 n}\right)$ (see the proof in the Appendix).

At $q=1$ the matrix $S(u)$ becomes $A-u^{-1} A^{t}$. Hence, the Sklyanin determinant sdet $S(u)$ becomes $\gamma(u) \operatorname{det}\left(A-u^{-1} A^{t}\right)$, where $\gamma(u)$ is defined in (3.5) with $N=2 n$. Therefore, replacing $u$ with $-\lambda^{-1}$ we thus prove that all coefficients of $\operatorname{det}\left(A+\lambda A^{t}\right)$ are Casimir elements for the Poisson bracket on $\hat{\mathcal{P}}_{2 n}$.

As in the orthogonal case, we have $f_{2 n-i}=f_{i}$ for all $i=0,1, \ldots, 2 n$. Note also that $f_{0}=f_{2 n}=\operatorname{det} A$ and so we have the following relation between the Casimir elements

$$
f_{0}=\prod_{k=1}^{n}\left(a_{2 k, 2 k} a_{2 k-1,2 k-1}-a_{2 k, 2 k-1} a_{2 k-1,2 k}\right) .
$$

Conjecture 4.4. The algebra of Casimir elements of $\hat{\mathcal{P}}_{2 n}$ is generated by the family of elements provided by Theorem 4.3 and the Pfaffian $\operatorname{Pf}\left(A-A^{t}\right)$. 
In the rest of this section we work with the twisted quantized enveloping algebra $\mathrm{U}_{q}^{\prime}\left(\mathfrak{s p}_{2 n}\right)$. Recall the action of the braid group $B_{2 n}$ on the quantized enveloping algebra $\mathrm{U}_{q}\left(\mathfrak{g l}_{2 n}\right)$; see Section 2 ,

Proposition 4.5. The subalgebra $\mathrm{U}_{q}^{\prime}\left(\mathfrak{s p}_{2 n}\right) \subset \mathrm{U}_{q}\left(\mathfrak{g l}_{2 n}\right)$ is stable under the action of the elements $\beta_{1}, \beta_{3}, \ldots, \beta_{2 n-1}$ of $B_{2 n}$.

Proof. Observe that the algebra $\mathrm{U}_{q}^{\prime}\left(\mathfrak{s p}_{2 n}\right)$ is generated by the elements

$$
s_{i i}, \quad s_{i+1, i+1}, \quad s_{i, i+1}, \quad s_{i, i+1}^{-1} \quad \text { for } \quad i=1,3, \ldots, 2 n-1
$$

and

$$
s_{i+3, i+1} \quad \text { for } \quad i=1,3, \ldots, 2 n-3 .
$$

Indeed, $s_{i+1, i}$ for odd $i$ can be expressed in terms of the elements (4.6) from (4.3). Furthermore, the remaining generators can be expressed in terms of the elements (4.6) and

$$
s_{i+2, i}, \quad s_{i+2, i+1}, \quad s_{i+3, i} \quad s_{i+3, i+1} \quad \text { for } \quad i=1,3, \ldots, 2 n-3
$$

by induction from the relations

$$
\left(q-q^{-1}\right) s_{k l}=s_{i, i+1}^{-1}\left(s_{k, i+1} s_{i l}-s_{i l} s_{k, i+1}\right), \quad k>i+1, \quad i>l, \quad i \text { odd }
$$

which are implied by the defining relations (2.10). However, for each $i$ as in (4.8) we have

$$
\begin{aligned}
\left(q-q^{-1}\right) s_{i+3, i} & =s_{i, i+1}^{-1}\left(s_{i+3, i+1} s_{i i}-s_{i i} s_{i+3, i+1}\right), \\
\left(q-q^{-1}\right) s_{i+2, i+1} & =s_{i+3, i+2}^{-1}\left(s_{i+3, i+1} s_{i+2, i+2}-s_{i+2, i+2} s_{i+3, i+1}\right), \\
\left(q-q^{-1}\right) s_{i+2, i} & =s_{i+3, i+2}^{-1}\left(s_{i+3, i} s_{i+2, i+2}-s_{i+2, i+2} s_{i+3, i}\right) .
\end{aligned}
$$

Hence, it suffices to verify that the images of the elements (4.6) and (4.7) under the action of $\beta_{1}, \beta_{3}, \ldots, \beta_{2 n-1}$ are contained in $\mathrm{U}_{q}^{\prime}\left(\mathfrak{s p}_{2 n}\right)$. These images can be explicitly calculated from (4.4). For any odd $j$ the elements (4.6) with $i \neq j$ are fixed by the action of $\beta_{j}$, while

$$
\beta_{j}: s_{j j} \mapsto s_{j, j+1}^{-2} s_{j+1, j+1}, \quad s_{j+1, j+1} \mapsto q^{-2} s_{j j}, \quad s_{j, j+1} \mapsto q^{2} s_{j, j+1}^{-1}
$$

Moreover, the elements (4.7) with $i \neq j-2, j$ are fixed by the action of $\beta_{j}$, while

$$
\beta_{j}: s_{j+1, j-1} \mapsto q^{-1} s_{j, j-1}, \quad s_{j+3, j+1} \mapsto q^{-1} s_{j+3, j}
$$

All these relations are verified by direct calculation with the use of the defining relations of $\mathrm{U}_{q}\left(\mathfrak{g l}_{2 n}\right)$. 
In particular, the restrictions of the action of $\beta_{1}, \beta_{3}, \ldots, \beta_{2 n-1}$ to the subalgebra $\mathrm{U}_{q}^{\prime}\left(\mathfrak{s p}_{2 n}\right)$ yield automorphisms of the latter.

Now observe that the elements $\gamma_{1}, \gamma_{3}, \ldots, \gamma_{2 n-3}$ of $B_{2 n}$ given by

$$
\gamma_{2 k-1}=\beta_{2 k} \beta_{2 k-1} \beta_{2 k+1} \beta_{2 k}, \quad k=1, \ldots, n-1
$$

generate a subgroup of $B_{2 n}$ isomorphic to $B_{n}$. The braid relations for the $\gamma_{2 k-1}$ are easily verified with the use of their geometric interpretation. Indeed, if we regard $\beta_{j}$ as the braid

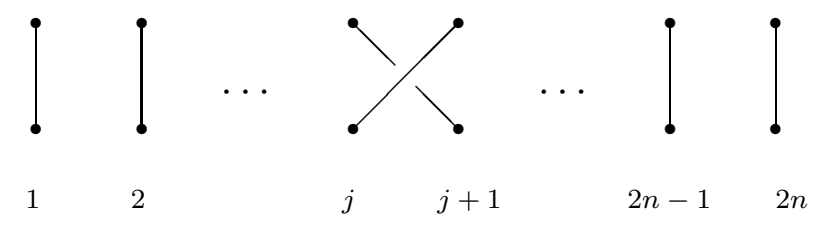

then each $\gamma_{2 i-1}$ is just an elementary braid on the doubled strands:

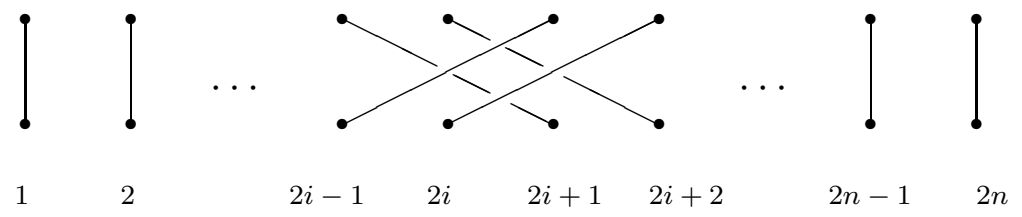

For each odd $i$ the elements (4.6) generate a subalgebra of $\mathrm{U}_{q}^{\prime}\left(\mathfrak{s p}_{2 n}\right)$ isomorphic to $\mathrm{U}_{q}^{\prime}\left(\mathfrak{s p}_{2}\right)$. The next proposition shows that the elements $\gamma_{i}$ permute these subalgebras.

Proposition 4.6. The images of the elements (4.6) under the action of the automorphisms $\gamma_{1}, \gamma_{3}, \ldots, \gamma_{2 n-3}$ belong to $\mathrm{U}_{q}^{\prime}\left(\mathfrak{s p}_{2 n}\right)$.

Proof. This is verified with the use of (4.4). For any odd $j$ the elements (4.6) with $i \neq j, j+2$ are fixed by the action of $\gamma_{j}$, while

$$
\gamma_{j}: s_{j j} \mapsto s_{j+2, j+2}, \quad s_{j+1, j+1} \mapsto s_{j+3, j+3}, \quad s_{j, j+1} \mapsto s_{j+2, j+3}
$$

and

$$
\gamma_{j}: s_{j+2, j+2} \mapsto s_{j j}, \quad s_{j+3, j+3} \mapsto s_{j+1, j+1}, \quad s_{j+2, j+3} \mapsto s_{j, j+1} .
$$

This follows from the formulas for the action of the $\beta_{i}$ on $\mathrm{U}_{q}\left(\mathfrak{g l}_{2 n}\right)$ which imply, for instance, relations of the type

$$
\beta_{j} \beta_{j+1}: t_{j+1, j} \mapsto t_{j+2, j+1}
$$

Since $\gamma_{j}=\beta_{j+1} \beta_{j+2} \beta_{j} \beta_{j+1}$, this gives $\gamma_{j}: t_{j+1, j} \mapsto t_{j+3, j+2}$. The images of the remaining elements of the form $t_{j j}, \bar{t}_{j, j+1}, t_{j+1, j+1}$ are calculated in a similar way which gives the desired formulas. 
It can be shown that Proposition 4.6 is not extended to the remaining generators (4.8) of the algebra $\mathrm{U}_{q}^{\prime}\left(\mathfrak{s p}_{2 n}\right)$. Observe that the elements $\beta_{i}$ and $\gamma_{i}$ of $B_{2 n}$ with odd $i$ satisfy the relations

$$
\gamma_{i}^{-1} \beta_{j} \gamma_{i}=\beta_{j} \quad \text { if } \quad j \neq i, i+2
$$

while

$$
\gamma_{i}^{-1} \beta_{i} \gamma_{i}=\beta_{i+2} \quad \text { and } \quad \gamma_{i}^{-1} \beta_{i+2} \gamma_{i}=\beta_{i} .
$$

The elements $\beta_{i}$ generate a subgroup of $B_{2 n}$ isomorphic to $\mathbb{Z}^{n}$. We shall identify $\mathbb{Z}^{n}$ with this subgroup. These observations suggest the following definition. Consider the braid group $B_{n}$ with generators $\gamma_{1}^{\prime}, \gamma_{3}^{\prime}, \ldots, \gamma_{2 n-3}^{\prime}$ and the usual defining relations

$$
\gamma_{i}^{\prime} \gamma_{i+2}^{\prime} \gamma_{i}^{\prime}=\gamma_{i+2}^{\prime} \gamma_{i}^{\prime} \gamma_{i+2}^{\prime}, \quad i=1,3, \ldots, 2 n-5
$$

and

$$
\gamma_{i}^{\prime} \gamma_{j}^{\prime}=\gamma_{j}^{\prime} \gamma_{i}^{\prime}, \quad|i-j|>2 .
$$

Define the group $\Gamma_{n}$ as the semidirect product $\Gamma_{n}=B_{n} \ltimes \mathbb{Z}^{n}$ where the action of $B_{n}$ on $\mathbb{Z}^{n}$ is defined by

$$
\beta_{j}^{\gamma_{i}^{\prime}}=\beta_{j} \quad \text { if } \quad j \neq i, i+2
$$

while

$$
\beta_{i}^{\gamma_{i}^{\prime}}=\beta_{i+2} \quad \text { and } \quad \beta_{i+2}^{\gamma_{i}^{\prime}}=\beta_{i} .
$$

Note that the Weyl group $W\left(C_{n}\right)=\mathfrak{S}_{n} \ltimes \mathbb{Z}_{2}^{n}$ of type $C_{n}$ may be regarded as a classical counterpart of $\Gamma_{n}$.

Conjecture 4.7. There exists an action of the group $\Gamma_{n}$ on the algebra $\mathrm{U}_{q}^{\prime}\left(\mathfrak{s p}_{2 n}\right)$ by automorphisms which corresponds to the action of $W\left(C_{n}\right)$ on $\mathrm{U}\left(\mathfrak{s p}_{2 n}\right)$.

Our final theorem shows that the conjecture holds for $n=2$.

Theorem 4.8. Let the generators $\beta_{1}$ and $\beta_{3}$ of the group $\Gamma_{2}$ act on $\mathrm{U}_{q}^{\prime}\left(\mathfrak{s p}_{4}\right)$ as in Proposition 4.5 and let the generator $\gamma_{1}^{\prime}$ act on the elements (4.6) with $i=1,3$ as $\gamma_{1}$. Then together with the assignment

$$
\gamma_{1}^{\prime}: s_{32} \mapsto s_{41}, \quad s_{41} \mapsto s_{32}, \quad s_{31} \mapsto s_{31}, \quad s_{42} \mapsto s_{42}
$$

this defines an action of $\Gamma_{2}$ on $\mathrm{U}_{q}^{\prime}\left(\mathfrak{s p}_{4}\right)$ by automorphisms.

Proof. It is easy to verify that $\gamma_{1}^{\prime}$ respects the defining relations of $\mathrm{U}_{q}^{\prime}\left(\mathfrak{s p}_{4}\right)$. For instance, the following relations are clearly respected by $\gamma_{1}^{\prime}$

$$
\begin{aligned}
& s_{33} s_{32}=s_{32} s_{33}, \quad s_{11} s_{32}=s_{32} s_{11}+\left(q^{-1}-q\right) s_{12} s_{31} \\
& s_{31} s_{32}=q^{-1} s_{32} s_{31}+\left(q-q^{-1}\right)\left(q^{-1} s_{21} s_{33}-s_{12} s_{33}\right)
\end{aligned}
$$


and

$$
\begin{aligned}
& s_{11} s_{41}=s_{41} s_{11}, \quad s_{33} s_{41}=s_{41} s_{33}+\left(q^{-1}-q\right) s_{34} s_{31} \\
& s_{31} s_{41}=q^{-1} s_{41} s_{31}+\left(q-q^{-1}\right)\left(q^{-1} s_{43} s_{11}-s_{34} s_{11}\right)
\end{aligned}
$$

together with

$$
s_{32} s_{41}=s_{41} s_{32}+\left(q-q^{-1}\right)\left(s_{12} s_{43}-s_{34} s_{21}\right),
$$

and this holds for the remaining relations as well. The defining relations of the group $\Gamma_{2}$ are also easily verified.

\section{Appendix}

Here we prove that the Sklyanin determinant sdet $S(u)$ is central in the extended algebra $\hat{\mathrm{U}}_{q}^{\prime}\left(\mathfrak{s p}_{N}\right)$ with $N=2 n$; see the proof of Theorem 4.3, We need to introduce some more notation. Following [19], introduce the trigonometric $R$-matrix

$$
\begin{aligned}
R(u, v) & =(u-v) \sum_{i \neq j} E_{i i} \otimes E_{j j}+\left(q^{-1} u-q v\right) \sum_{i} E_{i i} \otimes E_{i i} \\
& +\left(q^{-1}-q\right) u \sum_{i>j} E_{i j} \otimes E_{j i}+\left(q^{-1}-q\right) v \sum_{i<j} E_{i j} \otimes E_{j i}
\end{aligned}
$$

and a rational function in independent variables $u_{1}, \ldots, u_{r}, q$ valued in $\left(\text { End } \mathbb{C}^{N}\right)^{\otimes r}$ by

$$
R\left(u_{1}, \ldots, u_{r}\right)=\prod_{i<j} R_{i j}\left(u_{i}, u_{j}\right),
$$

where the product is taken in the lexicographical order on the pairs $(i, j)$. We have the following relation in the algebra $\hat{U}_{q}^{\prime}\left(\mathfrak{s p}_{N}\right) \otimes\left(\text { End } \mathbb{C}^{N}\right)^{\otimes r}$,

$$
\begin{aligned}
& R\left(u_{1}, \ldots, u_{r}\right) S_{1}\left(u_{1}\right) R_{12}^{t} \cdots R_{1 r}^{t} S_{2}\left(u_{2}\right) R_{23}^{t} \cdots R_{2 r}^{t} S_{3}\left(u_{3}\right) \cdots R_{r-1, r}^{t} S_{r}\left(u_{r}\right)= \\
& S_{r}\left(u_{r}\right) R_{r-1, r}^{t} \cdots S_{3}\left(u_{3}\right) R_{2 r}^{t} \cdots R_{23}^{t} S_{2}\left(u_{2}\right) R_{1 r}^{t} \cdots R_{12}^{t} S_{1}\left(u_{1}\right) R\left(u_{1}, \ldots, u_{r}\right)
\end{aligned}
$$

see [19], where $R_{i j}^{t}=R_{i j}^{t}\left(u_{i}^{-1}, u_{j}\right)$ with $R^{t}(u, v)$ defined in (3.4). Now take $r=N+1$ and label the copies of End $\mathbb{C}^{N}$ in the tensor product $\hat{\mathrm{U}}_{q}^{\prime}\left(\mathfrak{s p}_{N}\right) \otimes\left(\text { End } \mathbb{C}^{N}\right)^{\otimes(N+1)}$ with the indices $0,1, \ldots, N$. Furthermore, specialize the parameters $u_{i}$ in (5.3) as follows:

$$
u_{0}=v, \quad u_{i}=q^{-2 i+2} u \quad \text { for } i=1, \ldots, N
$$

Then by [19, Proposition 4.1], the element (5.2) will take the form

$$
R\left(v, u, \ldots, q^{-2 N+2} u\right)=\alpha(u) \prod_{i=1, \ldots, N}^{\longrightarrow} R_{0 i}\left(v, q^{-2 i+2} u\right) A_{N}^{q}
$$


where

$$
\alpha(u)=u^{N(N-1) / 2} \prod_{1 \leqslant i<j \leqslant N}\left(q^{-2 i+2}-q^{-2 j+2}\right) .
$$

We shall now be verifying that

$$
\prod_{i=1, \ldots, N} R_{0 i}\left(v, q^{-2 i+2} u\right) A_{N}^{q}=\delta(u, v) A_{N}^{q}
$$

where

$$
\delta(u, v)=\left(q^{-1} v-q u\right) \prod_{i=1}^{N-1}\left(v-q^{-2 i} u\right) .
$$

The $R$-matrix $R(u, v)$ satisfies the Yang-Baxter equation

$$
R_{12}(u, v) R_{13}(u, w) R_{23}(v, w)=R_{23}(v, w) R_{13}(u, w) R_{12}(u, v) .
$$

Using this relation repeatedly, we derive the identity

$$
R\left(u_{1}, \ldots, u_{r}\right)=\prod_{i<j} R_{i j}\left(u_{i}, u_{j}\right),
$$

where the product is taken in the order opposite to the lexicographical order on the pairs $(i, j)$. Taking here $r=N+1$ and specializing the variables $u_{i}$ as above, we arrive at

$$
\prod_{i=1, \ldots, N} R_{0 i}\left(v, q^{-2 i+2} u\right) A_{N}^{q}=A_{N}^{q} \prod_{i=1, \ldots, N}^{\overleftarrow{ }} R_{0 i}\left(v, q^{-2 i+2} u\right)
$$

Hence, for the proof of (5.4), it now suffices to compare the images of the operators on both sides at the basis vectors of the form $v_{k}=e_{k} \otimes e_{i_{1}} \otimes \cdots \otimes e_{i_{N}}$ with $k=$ $1, \ldots, N$, where the $e_{i}$ denote the canonical basis vectors of $\mathbb{C}^{N}$ and $\left\{i_{1}, \ldots, i_{N}\right\}$ is a fixed permutation of $\{1, \ldots, N\}$. Our next observation is the fact that for any $i, j \in\{1, \ldots, N\}$ the expression $R(u, v)\left(e_{i} \otimes e_{j}\right)$ is a linear combination of $e_{i} \otimes e_{j}$ and $e_{j} \otimes e_{i}$. This implies that for each $k$,

$$
A_{N}^{q} \prod_{i=1, \ldots, N}^{\overleftarrow{ }} R_{0 i}\left(v, q^{-2 i+2} u\right) v_{k}=\delta_{k}(u, v) A_{N}^{q} v_{k}
$$

for some scalar function $\delta_{k}(u, v)$ which is independent of the permutation $\left\{i_{1}, \ldots, i_{N}\right\}$. It remains to show that $\delta_{k}(u, v)=\delta(u, v)$ for all $k$. However, this is immediate from (5.6) if for a given $k$ we choose a permutation $\left\{i_{1}, \ldots, i_{N}\right\}$ with $i_{1}=k$, thus completing the proof of (5.4). 
Now apply the transposition $t$ on the 0-th copy of End $\mathbb{C}^{N}$ and combine (5.4) and (5.5) to derive another identity

$$
A_{N}^{q} \prod_{i=1, \ldots, N} R_{0 i}^{t}\left(v, q^{-2 i+2} u\right)=\prod_{i=1, \ldots, N}^{\longleftarrow} R_{0 i}^{t}\left(v, q^{-2 i+2} u\right) A_{N}^{q}=\delta(u, v) A_{N}^{q}
$$

Thus, (5.3) becomes

$$
\delta(u, v) \delta\left(u, v^{-1}\right) A_{N}^{q} S_{0}(v) \operatorname{sdet} S(u)=\delta(u, v) \delta\left(u, v^{-1}\right) A_{N}^{q} \operatorname{sdet} S(u) S_{0}(v),
$$

proving that sdet $S(u)$ lies in the center of $\hat{\mathrm{U}}_{q}^{\prime}\left(\mathfrak{s p}_{N}\right)$.

As a final remark, note that the above argument applies to more general matrices $S(u)$. The only property of $S(u)$ used above is the fact that $S(u)$ satisfies the reflection equation

$$
R(u, v) S_{1}(u) R^{t}\left(u^{-1}, v\right) S_{2}(v)=S_{2}(v) R^{t}\left(u^{-1}, v\right) S_{1}(u) R(u, v) .
$$

This implies that (3.3) equals $A_{N}^{q}$ sdet $S(u)$ for some formal series sdet $S(u)$ called the Sklyanin determinant. Then sdet $S(u)$ is central in the algebra with the defining relations (5.7). In particular, this applies to the (extended) twisted $q$-Yangians associated with the orthogonal and symplectic Lie algebras; see [19].

\section{References}

[1] P. P. Boalch, Stokes matrices, Poisson Lie groups and Frobenius manifolds, Invent. Math. 146 (2001), 479-506.

[2] A. I. Bondal, A symplectic groupoid of triangular bilinear forms and the braid group, Izvestiya: Mathematics 68 (2004), 659-708.

[3] A. I. Bondal, Symplectic groupoids related to Poisson-Lie groups, Proc. Steklov Inst. Math. 246 (2004), 34-53.

[4] V. Chari and A. Pressley, A Guide to Quantum Groups, Cambridge University Press, Cambridge, 1994.

[5] L. O. Chekhov, Teichmüller theory of bordered surfaces, preprint ITEP/TH53/06, math.AG/0610872.

[6] L. O. Chekhov and V. V. Fock, Observables in 3D gravity and geodesic algebras, Czechoslovak J. Phys. 50 (2000), 1201-1208. 
[7] N. Ciccoli and F. Gavarini, A quantum duality principle for coisotropic subgroups and Poisson quotients, Adv. Math. 199 (2006), 104-135.

[8] B. Dubrovin, Geometry of 2D topological field theory, in "Integrable systems and quantum groups" (M. Francaviglia, S. Greco, Eds), Lect. Notes. Math. 1620, Springer, 1996, pp. 120-348.

[9] D. B. Fairlie, Quantum deformations of SU(2), J. Phys. A 23 (1990), L183-L187.

[10] F. Gavarini, Presentation by Borel subalgebras and Chevalley generators for quantum enveloping algebras, Proc. Edinburgh Math. Soc. 49 (2006), 291-308.

[11] A. M. Gavrilik and A. U. Klimyk, q-deformed orthogonal and pseudo-orthogonal algebras and their representations, Lett. Math. Phys. 21 (1991), 215-220.

[12] A. M. Gavrilik and N. Z. Iorgov, On Casimir elements of q-algebras $U_{q}^{\prime}\left(\mathrm{so}_{n}\right)$ and their eigenvalues in representations, in 'Symmetry in nonlinear mathematical physics', Proc. Inst. Mat. Ukr. Nat. Acad. Sci. 30, Kyiv, 1999, pp. 310-314.

[13] N. Z. Iorgov and A. U. Klimyk, The nonstandard deformation $U_{q}^{\prime}\left(\mathrm{so}_{n}\right)$ for $q$ a root of unity, Methods of Funct. Anal. Topology 6 (2000), 15-29.

[14] N. Z. Iorgov and A. U. Klimyk, Classification theorem on irreducible representations of the q-deformed algebra $\mathrm{U}_{q}^{\prime}\left(\mathfrak{s o}_{n}\right)$, Int. J. Math. Sci. 2005, no. 2, 225-262.

[15] M. Itoh and T. Umeda, On central elements in the universal enveloping algebras of the orthogonal Lie algebras, Compos. Math. 127 (2001), 333-359.

[16] G. Lusztig, Finite-dimensional Hopf algebras arising from quantized universal enveloping algebras, J. Amer. Math. Soc. 3 (1990), 257-296.

[17] A. I. Molev, Representations of the twisted quantized enveloping algebra of type $C_{n}$, Moscow Math. J. 6 (2006), 531-551.

[18] A. Molev and M. Nazarov, Capelli identities for classical Lie algebras, Math. Ann. 313 (1999), 315-357.

[19] A. Molev, E. Ragoucy and P. Sorba, Coideal subalgebras in quantum affine algebras, Rev. Math. Phys. 15 (2003), 789-822.

[20] J. E. Nelson and T. Regge, $2+1$ quantum gravity, Phys. Lett. B 272 (1991), 213-216. 
[21] J. E. Nelson and T. Regge, $2+1$ gravity for genus $>$ 1, Comm. Math. Phys. 141 (1991), 211-223.

[22] J. E. Nelson and T. Regge, Invariants of $2+1$ gravity, Comm. Math. Phys. 155 (1993), 561-568.

[23] J. E. Nelson, T. Regge and F. Zertuche, Homotopy groups and (2+1)-dimensional quantum de Sitter gravity, Nucl. Phys. B 339 (1990), 516-532.

[24] M. Noumi, Macdonald's symmetric polynomials as zonal spherical functions on quantum homogeneous spaces, Adv. Math. 123 (1996), 16-77.

[25] M. Noumi, T. Umeda and M. Wakayama, Dual pairs, spherical harmonics and a Capelli identity in quantum group theory, Compos. Math. 104 (1996), 227-277.

[26] A. Odesskii, An analogue of the Sklyanin algebra, Funct. Anal. Appl. 20 (1986), $152-154$.

[27] A. Odesskii and V. Rubtsov, Polynomial Poisson algebras with regular structure of symplectic leaves, Theor. Mat. Phys. 133 (2002), 3-24.

[28] M. R. Santilli, A realization of the $U_{q, 1}$ algebra in terms of quantum-mechanical operators, Nuovo Cimento A (10) 51 (1967), 74-88.

[29] M. Ugaglia, On a Poisson structure on the space of Stokes matrices, Int. Math. Res. Notices 6 (1999), 473-493.

[30] Ping Xu, Dirac submanifolds and Poisson involutions, Ann. Sci. École Norm. Sup. 36 (2003), 403-430. 